\title{
Au cœur de la révolution burkinabè
}

Sten Hagberg, Ludovic Kibora, Fatoumata Ouattara et Adjara Konkobo

\section{(2) OpenEdition}

1 Journals

Édition électronique

URL : http://journals.openedition.org/anthropodev/499

DOI : 10.4000/anthropodev.499

ISSN : 2553-1719

Éditeur

APAD - Association pour l'anthropologie du changement social et du développement

\section{Édition imprimée}

Date de publication : 1 septembre 2015

Pagination : 199-224

ISBN : 979-10-93476-02-5

ISSN : 2276-2019

Référence électronique

Sten Hagberg, Ludovic Kibora, Fatoumata Ouattara et Adjara Konkobo, « Au cœur de la révolution burkinabè », Anthropologie \& développement [En ligne], 42-43 | 2015, mis en ligne le 01 décembre 2016, consulté le 03 mai 2019. URL : http://journals.openedition.org/anthropodev/499 ; DOI : 10.4000/anthropodev.499

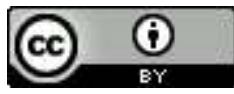

La revue Anthropologie \& développement est mise à disposition selon les termes de la Licence Creative Commons Attribution 4.0 International. 


\title{
Au cœur de la révolution burkinabè
}

\author{
Sten Hagberg, Ludovic Kibora, Fatoumata Ouattara \\ et Adjara Konkobo
}

Ce texte est le résultat d'un projet ethnographique conjoint pour narrer la révolution burkinabè de octobre-novembre 2014 sur la base des expériences vécues au-dedans et en dehors du soulèvement populaire. Il analyse les événements précédant la chute de Blaise Compaoré, les journées désormais historiques de la révolution, ainsi que les premiers mois de la transition politique. Le texte est basé sur la combinaison de l'enquête de terrain ethnographique (ici surtout l'observation participante), le suivi de mass médias et des réseaux sociaux, et l'analyse de l'histoire politique moderne burkinabè. En particulier, nous discutons comment la légalité et la légitimité s'articulent avec les processus fondamentaux du changement et de la continuité au Burkina Faso en pleine transition politique. Pour conclure, nous proposons le fait qu'il existe de vraies potentialités pour bâtir une démocratie à la fois légitime et légale.

This paper is the result of a joint ethnographic endeavor to narrate the Burkinabe revolution of October-November 2014 on the basis of lived experiences inside and outside of the popular uprising. It analyses the events that anteceded the Fall of President Blaise Compaoré, the days of revolution, as well as the first months of the oneyear political transition. The paper is based on the combination of ethnographic fieldwork of participant observation, careful monitoring of mass media and social media, and analysis of modern political history in Burkina Faso. Throughout the paper we particularly discuss how legality and legitimacy articulate with fundamental processes of change and continuity in a Burkina Faso in the midst of a political transition. In conclusion, we suggest that there are true potentialities to build a democracy that is both legal and legitimate. 


\section{Introduction}

En octobre-novembre 2014, le Burkina Faso a été au cœur du soulèvement et de l'insurrection populaires, aujourd'hui appelés "la révolution ». La proposition d'une loi modifiant l'Article 37 de la Constitution, qui aurait ouvert la voie pour une présidence à vie à Blaise Compaoré, a fait déborder le vase ${ }^{1}$. L'opposition politique et la société civile se mobilisèrent alors avec des pancartes telles que "Blaise dégage " et "Blaise=Ebola du Burkina » lors des manifestations des 27,28 et 29 octobre. Le 30 octobre les manifestants prirent d'assaut l'Assemblée nationale, puis la brûlèrent pour signifier avec force leur opposition à cette loi, déclenchant ainsi l'insurrection. Le 31 octobre, Blaise Compaoré démissionna après avoir passé vingt-sept ans au pouvoir.

Cet article $^{2}$ sur la révolution burkinabè est le fruit d'un travail collectif au plus près du terrain, qui vise à analyser les événements précédant la chute de Blaise Compaoré à partir d'observations faites au moment du soulèvement et de l'insurrection, puis lors des premiers pas de la transition. Il s'agira ici de voir comment les jeux de légalité et de légitimité se sont articulés pour décrire le processus par lequel "le système Compaoré ", manifestement puissant et bien ancré, en est arrivé là. Nous avons cherché à combiner l'observation participante avec un peu plus de recul analytique.

Une telle démarche "à chaud " est néanmoins vite confrontée à un problème de terminologie. Depuis le 30 octobre, des termes tels que " insurrection ", " soulèvement " et " révolution " sont beaucoup utilisés dans la presse et les réseaux sociaux ainsi que par une bonne partie de citoyens

\footnotetext{
${ }^{1}$ L'Article 37 stipule la durée du mandat et les modalités de l'éligibilité du président : « Le président est élu pour cinq ans. Il est rééligible une seule fois " (Constitution du Burkina Faso, 1991). Selon la proposition de loi, le texte aurait été modifié comme suit : "Le Président du Faso est élu pour cinq ans au suffrage universel direct, égal et secret. II est rééligible deux fois" ".

2 Nous remercions les lecteurs anonymes d'Anthropologie \& Développement pour des commentaires précieux et des critiques pointues de cette ethnographie " à chaud ".
} 
burkinabè et autres, et même lors de discours à caractère diplomatique ${ }^{3}$. Néanmoins, certains observateurs internationaux et nationaux parlent d'un " coup d'État » ou d'un " complot ». Ceci est surtout détectable parmi les militants de l'ancien parti présidentiel (le $\left.\mathrm{CDP}^{4}\right)$ et, peu étonnamment, chez les sympathisants de l'extrême gauche du parti clandestin PCRV ${ }^{5}$. Notre usage du terme "révolution " se fonde sur la terminologie utilisée par la plupart de nos interlocuteurs - approche émique - pour souligner le caractère populaire et dramatique de la rupture intervenue, sans pour autant s'inscrire dans un débat conceptuel et idéologique.

\section{Les précédents, les préparatifs et les tournants décisifs}

La révolution a été largement articulée autour des luttes de légitimité et de légalité. Ces luttes s'inscrivent dans une histoire politique burkinabè particulière, du soulèvement populaire contre le président Maurice Yaméogo le 3 janvier 1966, en passant par la Révolution démocratique et populaire de Thomas Sankara (1983-1987) et les mouvements contre les dossiers pendants de crimes de sang de l'ère Compaoré, jusqu'aux soulèvements de 2011. Ces luttes entre légalité et légitimité se sont aussi articulées à travers un certain légalisme politique - c'est-à-dire le légal instrumentalisé à des fins politiques - qui a contribué à consolider le pouvoir militaire de Blaise Compaoré. À la suite du coup d'État du 15 octobre 1987, un processus de démocratisation a été entamé en 1991. La construction de l'État de droit s'est alors basée sur la création d'institutions républicaines en même temps que tout était mis en œuvre pour qu'on ne puisse pas toucher au pouvoir du président. Une " démocratie à double façade " s'instaure avec d'une part la construction des institutions républicaines, c'est la façade

${ }^{3}$ Discours du président François Hollande lors du sommet de la Francophonie à Dakar le 29 novembre 2014.

${ }^{4}$ Le Congrès pour la Démocratie et le Progrès (CDP) est le parti au pouvoir qui a toujours accompagné Blaise Compaoré.

${ }^{5}$ Le Parti communiste révolutionnaire voltaïque (PCRV) est une force politique importante en ce qui concerne des mobilisations populaires et syndicales mais il ne participe pas aux élections (cf. Hagberg, 2010). 
extérieure (à l'image de celle d'un bâtiment), et d'autre part la tenue des élections présidentielles malgré les perceptions populaires d'un pouvoir à vie, c'est la façade en trompe l'œil (Hagberg, 2010). Cette démocratie à double façade a permis au président Blaise Compaoré de rester au-delà des débats politiques, évitant ainsi qu'on touche à son naam (" pouvoir » en mooré). Comme proposé par Mathieu Hilgers et Augustin Loada, le système Compaoré a joué sur l'incertitude des acteurs pour ce qui concerne les changements politiques : "Les réformes produisent systématiquement un espoir mais celui-ci est toujours quasi instantanément anéanti » (Hilgers et Loada, $2013:$ 199).

Blaise Compaoré a été élu lors des élections présidentielles de 1991, 1998, 2005 et 2010 respectivement, toujours avec une majorité écrasante. En 1991, le taux de participation était de $25 \%$, ce qui équivaut à un échec. Ces élections avaient été boycottées par les partis politiques de l'opposition comme le seront plus tard celles de 1998. En 2005, Blaise Compaoré s'est présenté comme " candidat nouveau ", suite à des changements constitutionnels intervenus en 1997 et en 2000. En novembre 2010, il obtient plus de $80 \%$ des votes au premier tour mais avec un taux de participation bas ${ }^{6}$. Aussi, dans les élections législatives, le Congrès pour la Démocratie et le Progrès - et son prédécesseur jusqu'en 1996, l'Organisation pour la Démocratie Populaire/Mouvement du Travail (ODP/MT) - a toujours fait de bons scores, parfois même des "chiffres soviétiques ». Lors des élections législatives en décembre 2012, le CDP obtient 70 des 127 sièges de l'Assemblée nationale, même si l'opposition fait des avancées significatives, notamment l'Union pour le Progrès et le Changement (UPC) de Zéphirin Diabré qui obtient 19 sièges et devient ainsi le chef de file de l'opposition politique.

En 2013, l'UPC mène les protestations contre la création du Sénat qu'il soupçonne d'être créé pour servir à modifier l'Article 37 et maintenir Blaise Compaoré au pouvoir (Paré, 2013). Avec un tiers des sénateurs nommés directement par le Président, le Sénat offre un beau potentiel pour ouvrir

\footnotetext{
${ }^{6}$ En 2010, le taux de participation était de $54,9 \%$, mais seules 3,2 millions de personnes étaient inscrites sur les listes électorales sur un potentiel de 7 millions d'électeurs (Ouestaf News, 2010).
} 
la voie à une présidence à vie. L'opposition politique et la société civile se mobilisent et les manifestations remplissent les rues dans les villes principales du pays. La tension monte, à tel point que Blaise Compaoré décide finalement de reporter la création du Sénat à une date ultérieure.

En janvier 2014, après des mois de rumeurs et de spéculations, trois architectes du "système Compaoré " - Roch Marc Christian Kaboré, Salif Diallo et Simon Compaoré - démissionnent du parti au pouvoir, suivis de nombreux autres dirigeants du CDP, pour former un nouveau parti, le Mouvement du Peuple pour le Progrès (MPP). Dans leur lettre de démission, ils protestent contre :

" des tentatives d'imposer la mise en place du Sénat aux forceps et [à] des velléités de réviser la Constitution dans le but de sauter le verrou de la limitation des mandats présidentiels dans un contexte où le peuple est profondément divisé » (Kaboré et al., 2014).

Cette démission annonce que 2014 sera une année tourmentée susceptible d'ouvrir la voie à une fin du régime (Hagberg, 2014).

Le contexte social aussi a changé : les organisations de la société civile se sont rajeunies ces dernières années. Le Mouvement Burkinabè des Droits de l'Homme et des Peuples (MBDHP), si fort pendant et après l'affaire Norbert Zongo ${ }^{7}$, n'a pas été à l'avant-garde cette fois-ci (Frère, 2010 ; Luning, 2010). Par contre le Balai citoyen - une association fondée en 2013 - a joué un rôle central à côté du Front de résistance citoyenne, du Collectif anti-référendum, du mouvement Ça suffit, des mouvements de jeunes et de femmes, etc. Ces mouvements étaient aussi très actifs sur les réseaux sociaux, avec des mises à jour à la minute sur Facebook et Twitter.

Le dernier recours de Blaise Compaoré consistait à proposer l'organisation d'un référendum sur l'Article 37, avec comme argumentaire que "le peuple » est souverain et que seul « le peuple " peut décider de modifier la Constitution. Face à ce mouvement général de protestation, il y

\footnotetext{
${ }^{7}$ Le journaliste et directeur de publication Norbert Zongo, qui avait à plusieurs reprises publié des articles critiques vis-à-vis du régime, a été sauvagement assassiné en décembre 1998. Les agents du régiment de la sécurité présidentielle furent les " sérieux suspects » de cet assassinat (Hagberg, 2002 ; Frère, 2010).
} 
avait le CDP et ses alliés tels que la Fédération Associative pour la Paix et le Progrès avec Blaise Compaoré (FEDAP/BC) et les partis de la mouvance présidentielle. Ces derniers se sont organisés à travers le Front républicain pour aider à changer la Constitution, en s'opposant aux partis politiques comme le MPP, I'UPC et le PDS/Metba ${ }^{8}$ ainsi qu'aux organisations de la société civile mobilisées contre la révision de l'Article 37. Des négociations entre l'opposition et Blaise Compaoré s'amorcent à la fin du mois de septembre 2014 sans que les deux camps ne parviennent à se mettre d'accord. Ces négociations sont aussi marquées par le décès inattendu du président du PDS/Metba, Hama Arba Diallo, maire de Dori et député à l'Assemblé nationale. Ce dernier s'était illustré par le " carton rouge " qu'il avait symboliquement brandi contre le président Blaise Compaoré lors d'un meeting gigantesque de l'opposition dans le plus grand stade de football de Ouagadougou en mai 2014 (Sanfo, 2014). Après l'insurrection du 30 octobre 2014, certains ont même proposé que Hama Arba Diallo soit le premier martyr de la révolution. En somme, cette lutte discursive entre légalité et légitimité a fini par plonger le pays dans une impasse où l'avenir s'annonçait fragile.

\section{Au cœur de l'insurrection}

À partir de divers sites d'observation ethnographique, en tant que manifestants, en tant qu'observateurs sur place et dans des réseaux sociaux et en tant que chercheurs engagés dans ce qui suit, nous tentons de dresser une chronologie de la révolution burkinabè. Mardi 21 octobre, un conseil des ministres extraordinaire décide d'introduire le projet de loi de modification de la Constitution à l'Assemblée nationale. Dans l'esprit de l'Article 161 et suivant de la Constitution, décision est prise, par le gouvernement, de procéder à la modification de l'Article 37 par un vote des députés ou par l'organisation d'un referendum si les trois quarts des voix ne sont pas acquises. La mouvance présidentielle (CDP et partis alliés) compte alors 81 députés favorables à la modification (AIB, 2014a). Le parti Alliance pour la Démocratie et la Fédération/Rassemblement Démocratique Afri-

${ }^{8}$ Parti pour la démocratie sociale/Parti des bâtisseurs. 
cain (ADF/RDA), qui clame depuis plusieurs mois son opposition à une telle modification, se déclare soudainement favorable (Paré, 2014). Ce parti compte 18 députés dont les voix sont précieuses pour faire passer cette loi. Par rapport à ce revirement, la rumeur ouagalaise fait état de corruption à coup de milliards de francs promis au président de I'ADF/RDA. La société civile crie au " coup d'état constitutionnel » et appelle à la " désobéissance civile ". La rumeur d'une crise politique et sociale imminente circule au sein de la capitale. Les uns et les autres conseillent à leurs proches de faire des provisions de vivres pour les jours à venir. Certaines personnes en rient, d'autres prennent cela au sérieux. Les prix de certaines denrées flambent. On sent qu'un événement se prépare.

\section{La marche des femmes}

Lundi 27 octobre, le Collectif des Femmes pour la Défense de la Constitution (COFEDEC) appelle à une marche contre la modification de l'Article 37. Des centaines de ouagalaises sortent avec des spatules levées et des balais pour entamer la semaine de désobéissance civile proclamée par les partis d'opposition et les représentants de la société civile. Cette manifestation est chargée de symbolique et de nombreux burkinabè nous ont confié que " quand les femmes sortent, là, c'est fini pour Blaise ".

Selon les représentations populaires, les femmes sont craintes car perçues comme des " êtres redoutables " et les malédictions qu'elles portent à un individu seraient suivies d'effets. La marche des femmes à Ouagadougou, ce jour, se présente comme une introduction dans cette plage de contestation politique. Les spatules sont utilisées pour la préparation du tô, la pâte de sorgho, de mil ou de maïs qui constitue le plat principal de plusieurs sociétés ouest-africaines. Notons aussi que certains militants du Balai citoyen y prennent activement part. Ce mouvement revendique une volonté de nettoyage de la malgouvernance et a choisi l'image du balai comme symbole de sa lutte citoyenne. Le balai est utilisé pour dégager les ordures/souillures du cadre de vie. L'objectif selon les initiateurs est d' " assainir citoyennement et proprement le Faso ».

Quant à l'image des femmes brandissant leurs spatules, elle marque, à ce moment précis des événements, fortement les esprits. Jamais dans l'histoire du pays, on n'avait assisté à une marche d'opposition féminine à 
l'égard d'un pouvoir politique. Certes, les femmes ont souvent pris part à des manifestations, mais leur propension à la contestation du pouvoir politique sur la place publique reste, dans la société burkinabè, tout de même marginale. Bien que la réalité soit tout autre, un imaginaire collectif continue de confiner les femmes dans les sphères de la reproduction et de l'accomplissement des tâches ménagères. Ainsi, la sortie des femmes dans " la rue " est perçue comme une remise en question de l'ordre établi : les femmes montrent le caractère inique de l'expression moaga consacrée " paaga la yiri » (la place de la femme c'est la maison). En brandissant sur la place publique l'instrument de l'espace domestique et privé (la spatule), les femmes montrent aux puissants que l'instrument nourricier se transforme en bâton expiatoire. La spatule ainsi levée au ciel devient une arme symbolique redoutée des hommes. Selon les représentations populaires, un homme qui recevrait un coup de spatule risquerait l'impuissance. Fortes de cette crainte, les forces de l'ordre n'auraient pas voulu s'opposer aux femmes, d'autant qu'elles étaient aussi considérées comme des (leurs) mères.

En même temps, cette marche des femmes était pour le moins inattendue puisque les organisations féminines devaient leur existence à la bénédiction du pouvoir de Blaise Compaoré. Alors, pour cette marche du 27 octobre, les femmes ont tenu à exprimer leur mécontentement et leurs craintes, mettant en garde Blaise Compaoré contre un bain de sang imminent. Face aux tensions suscitées par l'entêtement du Président à réviser la Constitution, elles espéraient lui signifier qu'il pouvait encore revenir sur sa décision. Elles lui donnaient encore une chance pour une sortie honorable et lui demandaient d'écouter, de ne pas s'entêter à vouloir rester au pouvoir en « truquant » les règles du jeu (Frintz, 2014).

Après la marche, les femmes étaient encore présentes dans les manifestations des 28,29 et 30 octobre, mais cette participation féminine a très peu été relayée et analysée par la presse. Par exemple, dans un article publié par le quotidien français Le Monde, "Six jours qui ont changé le Burkina Faso " (Barthet, 2014), il n'a été fait aucune mention du rôle joué par les femmes burkinabè. La presse nationale et internationale a plutôt mis en évidence les manifestations populaires des 28,30 et 31 octobre. Au niveau local, après quelques courts articles sur la " marche des spatules", il a fallu attendre le 27 novembre pour qu'un article de presse mette en 
évidence le témoignage d'une femme (Cendrine Nama) - artiste musicienne - affublée du titre de "héros de l'insurrection populaire $\left(n^{\circ} 9\right)$ " (Gue, 2014). Quant à la marche des femmes, elle témoigne :

"Quelle ne fut pas ma fierté de voir que bon nombre de ces femmes étaient des mères voir(e) des grand-mères d'un certain âge sorties pour leur peuple, pour leurs enfants parce que c'en était devenu trop. A $16 \mathrm{~h}$ nous avons entamé la marche spatules brandies scandant des phrases comme : 'touche pas à ma constitution'. "

En manifestant sans autorisation municipale le 27 octobre, les femmes ont bravé les cordons de policiers et de la compagnie républicaine de sécurité (CRS) qui avaient été placés pour leur barrer la route. C'était en réalité la première désobéissance civile, celle indiquant au reste du peuple que tout était désormais possible.

\section{La marche du 28 octobre}

L'appel à manifester le mardi 28 octobre est entendu par la population. Avant cette date, juchés sur des camions porte-char, les leaders de la société civile, notamment ceux du Balai citoyen, sillonnent les artères de la ville en invitant les gens à sortir massivement le jour J. Le Collectif antireferendum (CAR), le Front de Résistance citoyenne, etc., toutes ces organisations font un travail de mobilisation intense sur le terrain, aux côtés des partis politiques. Dans les bars et les buvettes, la révision de l'Article 37 est au centre de tous les débats.

Le 28 octobre, la mobilisation est historique. De nombreuses personnes estiment que le fait de marcher ce jour-là est une façon d'accomplir un devoir citoyen. En ralliant le lieu du rassemblement, c'est une ville morte qui s'offre à notre regard. Jeunes et vieux, hommes et femmes, la foule converge dès 7 heures du matin vers la place de la Nation. La plupart des manifestants sont à pied ou en mobylette. La marche prévue pour démarrer à 8 heures semble impossible à mettre en route tant la foule est immense. Des gens arrivent de tous les quartiers de la ville, individuellement ou par petits groupes. II y a des hommes et femmes du troisième âge mais aussi de très jeunes gens. En ce jour ouvrable, de nombreux services de l'administration publique et privée sont fermés. Euphorique, Zéphirin Diabré, le chef de file des partis d'opposition, dont le discours est difficilement 
audible à cause du brouhaha, déclare: "Cette fois-ci, ça passe ou ça casse ! " À nos côtés, un fonctionnaire à la retraite raconte avec joie qu'il n'avait pas manifesté contre le pouvoir en place depuis le 3 janvier 1966, lors de la chute du président Maurice Yaméogo. À la fin des allocutions, chacun repart chez lui avec le sentiment d'une mission bien accomplie.

Cette mobilisation, jamais égalée, aurait pu servir d'avertissement au président Blaise Compaoré. C'est ce qu'à ce stade des événements, les partisans d'un changement en douceur espéraient. Mais la surprise fût d'autant plus grande que, le soir, le ministre de l'Information, par ailleurs porte-parole du gouvernement, faisait passer un communiqué dans lequel il saluait la discipline observée par les marcheurs et concluait au dynamisme de la démocratie burkinabè (Traoré, 2014). Le lendemain, le Premier ministre demande à tous ses ministres de dresser une liste des fonctionnaires absents à leur poste le jour de la marche, pour d'éventuelles sanctions. Ceux qui espéraient, comme nous, que cette mobilisation guiderait le pouvoir en place vers une position de sagesse favorable au retrait du projet de loi, étaient très déçus. Les partisans de Blaise Compaoré prirent avec arrogance les agissements de ceux qui étaient contre la modification de l'Article 37. Dans une boutique, un employé, l'air inquiet, nous informe que de nombreux expatriés sont venus faire leurs provisions pour les jours à venir. Un client qui suivait la conversation lance: "Les gens ont peur, mais il n'y aura rien!" De tels propos et attitudes énervent même les citoyens les plus timorés. On a l'impression que tout le monde voit venir la catastrophe, sauf le pouvoir et ses affidés. Les acteurs du pouvoir en place semblent englués dans une surdité terrifiante à l'égard des voix contestant la légitimité de leur pouvoir. Au lieu de répondre aux attentes des marcheurs, le régime Compaoré développe d'autres stratégies pour le passage en force du projet de modification de la Constitution. Le même soir, les députés acquis à sa cause sont logés, pour ne pas dire séquestrés, à l'Hôtel Azalaï Indépendance. À partir de cet hôtel, ils peuvent ainsi joindre, par l'arrière-cour, l'Assemblée nationale et voter allègrement. À la radio, le président de l'Assemblée nationale, Soungalo Apollinaire Ouattara, fait passer un communiqué : I'heure du vote de la loi initialement fixée à 16 heures est ramenée à 10 heures. Rien que des stratégies sécuritaires. Mais cette attitude du pouvoir réussit plutôt à fédérer les différentes oppositions de tout bord contre lui. Le 29 octobre, les associations et syndi- 
cats de travailleurs, regroupés au sein de la Coalition contre la vie chère (CCVC), marquent leur désapprobation de la modification de l'Article 37. Pour une fois, partis politiques, organisations de la société civile et organisations syndicales sont tous unis contre le pouvoir avec, derrière eux, des centaines de militaires et de policiers radiés suite aux mutineries de 2011 et les victimes des licenciements liés à la privatisation d'entreprises publiques sous l'ère Compaoré.

Parallèlement, les fidèles du régime Compaoré distribuent des teeshirts estampillés " oui au référendum » à leurs militants et préparent la fête pour l'après-midi du 30 octobre. Pourtant, dans la ville, la menace de l'explosion est imminente : le 29 au soir la tension est palpable, les jeunes se préparent en brûlant des pneus pour obstruer les voies. Les SMS et messages Facebook font état de nombreuses échauffourées entre policiers et manifestants dans la ville de Ouagadougou. L'espoir d'un retrait du projet de loi à la dernière minute s'amenuise d'heure en heure. Blaise Compaoré et son régime restent toujours imperturbables. Les forces de l'ordre quadrillent le centre-ville durant toute la nuit. Face à la détermination du peuple, ce sera en vain.

\section{La prise de l'Assemblée nationale le 30 octobre}

Le jour du vote de la loi, des barrières de sécurité sont érigées à une distance de 1000 et 2000 mètres sur tous les axes qui mènent à l'Assemblée nationale. Le dispositif est impressionnant : policiers, gendarmes et gardes de la sécurité présidentielle. À moto ou à pied, les manifestants affluent par petits groupes vers les barrières de sécurité. Sur l'avenue Kwame $N^{\prime}$ Krumah, devant le siège du parti au pouvoir, des jeunes gens préparent la fête, sous la surveillance d'agents de la compagnie républicaine de sécurité. Le pouvoir ne semble pas avoir véritablement pris la mesure de la situation. Pendant ce temps, nous tentons de rallier l'Assemblée nationale par l'Est car la place de la Nation est déjà en ébullition. Les "antireferendum ", dont les rangs ne cessent de grossir, optent pour la stratégie offensive en jetant des projectiles sur les forces de police. Les manifestants sont bien préparés : les jeunes enduisent leur corps de beurre de karité ou d'huile de moteur, bouchent leur nez avec des morceaux d'étoffes imbibés d'eau, pour résister aux gaz lacrymogènes des policiers. La stratégie finit par payer. Ils ouvrent plusieurs fronts, à tel point que les forces de l'ordre 
sont débordées. On entend les premiers tirs. La radio Omega FM, que nous écoutons sur téléphonie portable ${ }^{9}$, donne la situation de la manifestation sur tous les fronts. Aux environs de 9h20, soit une demi-heure avant le vote de la "loi scélérate ", la foule franchit les portes de l'Assemblée nationale. Les forces de sécurité sont obligées de se consacrer à l'exfiltration des députés de la majorité alors que certains policiers préfèrent se fondre eux-mêmes dans la foule pour disparaître. L'Assemblée nationale est saccagée puis mise à feu.

Le gouvernement paniqué fait une déclaration pour retirer le projet de loi. C'est trop tard. Les manifestants sont déterminés à en finir avec le pouvoir et ses proches. Tous les députés de la majorité ont la vie sauve, même si quelques-uns ont pu être malmenés au passage. La plupart seront transférés dans un camp de la gendarmerie nationale non loin de là, pour leur sécurité. C'est alors qu'on constate que le résultat des manifestations a dépassé les attentes. L'émotion se lit sur les visages. Certains versent des larmes de joie, d'autres pleurent leurs camarades tombés sous les balles. Pour les manifestants, le pouvoir appartient désormais à " la rue ». Chaque quartier de la ville a ses barricades. Des mots d'ordre sont lancés par n'importe qui. La foule s'en prend à l'Azalaï Hôtel Indépendance où les députés ont passé leur dernière nuit avant le vote. Tout est saccagé. Puis cap est mis sur la Télévision nationale du Burkina accusée de diffuser des reportages très partisans et de ne pas couvrir l'insurrection populaire. Après l'atteinte de l'objectif premier, qui était de prendre l'Assemblée nationale, des groupes de manifestants, partout dans la ville, s'en prennent à des symboles du parti au pouvoir et pillent les domiciles, commerces et industries de certains proches du régime Compaoré. Un groupe de manifestants saccage et brûle le siège du CDP.

À la radio, Zéphirin Diabré, le chef de file de l'opposition politique, fait une déclaration dans laquelle il parle d'une dizaine de morts. Mais les manifestants exigent la démission du président Blaise Compaoré. L'opposition politique, qui n'avait pas inclus cette donne dans son agenda immédiat, est obligée de suivre et Zéphirin Diabré fait une autre déclaration en ce sens.

${ }^{9}$ http://tunein.com/radio/Radio-Omega-FM-Ouaga-1039-s201644/ 
Les manifestants convergent vers le palais présidentiel de Kosyam dans le quartier huppé de Ouaga 2000. Là encore, les forces de sécurité tirent à balles réelles, tuant deux manifestants, avant que des pourparlers ne s'engagent. Trois représentants des manifestants rencontrent le président Blaise Compaoré. Entretemps, tout le monde semble être pris au dépourvu. La rue dicte désormais sa loi. En rentrant en fin de matinée, nous croisons de nombreuses personnes rapportant "leurs butins de guerre " à la maison. La notion de biens mal acquis ne s'applique pas ici. La révolte contre le vote du projet de loi est en train de se muer en révolution contre une dictature. La télévision nationale étant hors d'usage, c'est la télévision privée Canal 3, considérée par le pouvoir comme favorable à l'opposition, qui va servir de canal au président Blaise Compaoré pour appeler au calme et au dialogue. Il promet de se retirer du pouvoir à la fin de son mandat.

\section{Le 31 octobre, la rue oblige Blaise Compaoré à quitter le pouvoir}

Manifestement, la déclaration faite par Blaise Compaoré la veille n'a pas été acceptée. Elle est contestée. Alors, loin de calmer la tension, cette sortie amène le peuple à se mobiliser davantage dès 8 heures, à la place de la Nation, désormais rebaptisée " place de la Révolution ", comme l'avait fait il y a une trentaine d'années Thomas Sankara. De nombreuses personnes sont massées devant l'État-major de l'armée à quelques mètres de là, tandis que nous apprenons que d'autres plus téméraires ont déjà pris le chemin du palais présidentiel pour "déloger Blaise Compaoré » et pour "libérer Kosyam ", comme le scandent les slogans. Dans les rues de Ouagadougou, on constate une absence totale des forces de l'ordre. Les pillages se poursuivent. La foule de militants massés dans la rue donne un ultimatum : à 12 heures Blaise Compaoré doit démissionner. À ce stade, dans la valse d'hésitations, les partis politiques n'ont pas de stratégie précise et commune pour la prise du pouvoir. Ils semblent dépassés par les mouvements de la société civile les plus actifs. À leurs yeux, il est important que l'armée prenne ses responsabilités pour garantir la stabilité et la sécurité dans le pays. À l'intérieur de l'État-major de l'armée il y a donc des tractations entre officiers supérieurs de l'armée et un ballet incessant de personnalités, notamment des leaders de la société civile. La radio nous informe que Blaise Compaoré a signé sa lettre de démission. La nouvelle 
est portée aux manifestants à 13 heures. Au même moment, la radio privée Omega FM, qui avait eu l'ingénieuse idée de placer des reporters dans tous les points chauds de la ville, annonce que le président Blaise Compaoré est parti dans un long cortège en direction de la ville de Pô ${ }^{10}$. Nous apprenons par la suite qu'il est sauvé en rase campagne par un hélicoptère de l'armée française, car sur le trajet de sa destination initiale, la population avait déjà commencé à lui barrer la route. C'est la liesse dans la ville de Ouagadougou. Coups de sifflets et de klaxons retentissent partout. Le chef d’État-major général des armées, le général Honoré Nabéré Traoré, qui avait annoncé, la veille, la dissolution du gouvernement et celle de l'Assemblée nationale, déclare assumer les "responsabilités de chef de l'Etat ". Mais sa proximité avec le président déchu et ses hésitations de la veille révoltent la foule. Dans l'après-midi, le lieutenant-colonel Yacouba Isaac Zida s'autoproclame, par la presse, à son tour chef de l'État en déclarant caduque toute disposition antérieure. La confusion règne. La foule qui, dès le premier jour, avait réclamé à cor et à cri le général à la retraite Kouamé Lougué, officier populaire auprès de la troupe et des civils, scande de nouveau son nom. Cependant, il y a bien longtemps que ce général a été éloigné de sa base par ses nominations administratives. L'impression que l'on a, c'est que le lieutenant-colonel Zida, le numéro 2 du régiment de la sécurité présidentielle (RSP), qui a le soutien de la société civile, s'impose à tous et l'armée finit par le reconnaître comme étant son représentant légitime désigné. La révolution vient de triompher et la sécurité du pays est confiée à l'armée. Dans les rues, les débits de boisson, qui sont restés fermés pendant deux jours, commencent à rouvrir leurs portes. Les commentaires vont bon train sur les événements en cours. On a l'impression qu'il n'y a jamais eu de partisans de la modification de l'Article 37.

10 Ville située à $147 \mathrm{~km}$ au Sud-est de la capitale, où se trouve le centre national d'entrainement commando où sont formés les éléments du régiment de la sécurité présidentielle. C'est de cette base que Blaise Compaoré est parti pour faire triompher la Révolution et porter au pouvoir d'abord Jean-Baptiste Ouédraogo en 1982 et ensuite Thomas Sankara en 1983. 
La confusion du 1er au 2 novembre et le début de la transition...

Dans la soirée du 31 octobre, les leaders des partis politiques et de la société civile appellent la population à arrêter tout acte de vandalisme et à sortir nombreux le samedi $1^{\text {er }}$ novembre pour une opération mana-mana ${ }^{11}$ (ville propre en dioula). Ouagadougou retrouve son visage même si elle porte encore des stigmates des manifestations. Dimanche 2 novembre est une journée confuse pendant laquelle les forces de l'ordre tuent un manifestant (Carayol et Roger, 2014), car " la rue » cherche son président civil pour éviter " la confiscation » de la révolution. A la télévision nationale, de violentes confrontations ont lieu après que le général à la retraite Kouamé Lougué se soit autoproclamé chef de l'État (sans que la déclaration ne soit passée sur les ondes), puis que le leader d'opposition politique Saran Sérémé ait été emmené par la foule pour faire, à son tour, une déclaration dans le même sens. Dans l'après-midi du 2 novembre, le lieutenant-colonel Zida rencontre les représentants des partis politiques. Ce moment marque le début de la transition ${ }^{12}$.

À partir du lundi 3 novembre, des personnalités dépêchées par la communauté internationale (ONU, UA et CEDEAO) arrivent à Ouagadougou. La pression de la rue, en conjonction avec celle de la communauté internationale, pèse sur les négociations pour élaborer une Charte de la transition (Charte de la Transition, 2014). Selon cette Charte, le président de la transition ne sera pas éligible pour les élections présidentielles en 2015. Tel sera aussi le cas des membres du gouvernement de la transition

${ }^{11}$ Mana-mana signifie dans la langue Dioula "faire briller, rendre propre ». Pendant le règne du président Thomas Sankara, c'était l'expression consacrée pour appeler les populations à assainir régulièrement leur cadre de vie. Opération Mana-mana devient ainsi une expression symbolisant les activités collectives de nettoyage des espaces publics.

12 Selon les estimations d'un comité ad hoc, 19 personnes sont mortes lors de l'insurrection populaire et 625 ont été blessées. Et 260 bâtiments privés et 14 bâtiments publics ont été saccagés. Cinq prisonniers sont morts à la Maison d'arrêt et de correction de Ouagadougou lors d'une tentative d'évasion le 30 octobre. Les dégâts matériels sont estimés à plus de 7,2 milliards de francs CFA (Loungé, 2014). Pour les pertes de vies humaines, le nombre annoncé ci-dessus a été modifié par la Coordination des structures pour l'assistance et le secours populaire (CAASP) qui a indiqué qu'il y a eu 33 morts (Kaboré et Ouédraogo, 2014). 
et du président du Conseil national de la transition, assemblée législative forte de 90 membres.

Le 16 novembre, Michel Kafando, ancien ministre des Affaires étrangères (1982-1983) et ambassadeur auprès des Nations unies (1998-2011), est sélectionné comme président de la transition par un collège spécial. Alors qu'il vient de rendre le pouvoir présidentiel, le lieutenant-colonel Zida est nommé premier ministre par le président Kafando. Outre le fait que Kafando ait été proposé par l'armée, Zida devient donc son premier ministre. Mais, étant donné tout ce que le peuple burkinabè a subi jusqu'ici, la situation est acceptée. Tout le monde est pressé de tourner la page de l'ère Compaoré, quelle que soit la voie choisie. Le 23 novembre, le Premier ministre annonce la composition du gouvernement de la transition. Le Conseil national de la transition (CNT) tient sa première session le 27 novembre et Chériff Sy, directeur de publication du quotidien Bendré, est désigné comme président. La révolution a alors abouti à un semblant de vie constitutionnelle à peu près normale ${ }^{13}$.

\section{Une révolution entre légalité et légitimité}

Cette ethnographie "à chaud", au cœur de l'insurrection, se base sur l'observation participante ou peut-être, au mieux, la " participation observante ". A travers nos observations, il est possible d'analyser comment des luttes entre légalité et légitimité se sont articulées au cœur de cette révolution. Un fil rouge qui ressort de cette ethnographie est que la révolution burkinabè de 2014 était une défense de la loi fondamentale (la Constitution de la $4^{\text {ème }}$ République) contre une récupération politique et politicienne.

\section{La désobéissance civile comme espace légitime}

C'est lors d'un conseil des ministres extraordinaire, le 21 octobre 2014, que le président Blaise Compaoré a franchi le Rubicon avec la décision de sou-

${ }^{13}$ Des analyses récentes de la révolution burkinabè ont d'ores et déjà été être publiées, notamment celles de Frère et Englebert (2015) et de Chouli (2015). 
mettre à l'Assemblée nationale la proposition de loi modifiant l'Article 37 de la Constitution. La mobilisation contre cette décision s'est faite rapidement et les réseaux sociaux ont été inondés par des propos d'indignation. Lorsque Gilbert Noël Ouédraogo, le président de l'ADF/RDA, a déclaré son soutien à la modification - contrairement à ses propos antérieurs - on ne parlait plus de referendum mais plutôt de faire voter la loi avec trois quarts des voix directement à l'Assemblée nationale. Alors, il y avait une réelle possibilité que la loi passe, ouvrant la voie à une présidence à vie. Dès le 22 octobre, l'opposition politique a lancé la campagne de " désobéissance civile " contre " un coup d'État constitutionnel » (AIB, 2014b). Cette désobéissance ouvrait un espace politique légitime nécessaire lorsque la loi ne peut plus s'appliquer.

La légitimité a aussi primé sur la légalité lorsque, le 27 octobre, des femmes (sans autorisation légale) sont sorties pour marcher contre Blaise Compaoré avec des spatules en main. Et le mardi 28 octobre, jour ouvrable, des centaines de milliers de personnes ont abandonné leurs emplois pour manifester dans les rues de Ouagadougou, comme pour donner un dernier avertissement à Blaise Compaoré. En voulant jouer sur la légalité, le Premier ministre a demandé à chaque ministre de sanctionner les fonctionnaires absents de leur poste ce jour-là. Le 29 octobre, autre jour ouvrable, les syndicats ont marché contre la vie chère et aussi contre la loi proposée. Le message émis lors de ces manifestations était clair : le projet de loi pouvait bien être légal mais il lui manquait toute légitimité. Au lieu de retirer la loi, le régime a pris la décision de sécuriser le vote en hébergeant tous les députés de la majorité à proximité immédiate de l'Assemblée nationale ${ }^{14}$. L'abus de l'usage de la légalité a été contré par la légitimité populaire qui avait fini par en avoir marre d'un tel jeu.

\section{La révolution et le pillage}

Jeudi 30 octobre, les manifestants ont pris d'assaut l'Assemblée nationale et l'insurrection s'est vite propagée à travers la ville. Les attaques avaient des cibles précises : le siège du CDP, l'Azalaï Hôtel Indépendance, les domi-

${ }^{14}$ Des sommes importantes - le chiffre de cinq millions de francs CFA circulait - auraient également été promises à chaque député de la majorité. 
ciles de François Compaoré, Alizéta Gando, Assimi Kouanda, Soungalo Ouattara et d'autres dignitaires du régime. Cependant, il y a aussi eu des saccages et pillages généralisés - c'est-à-dire des débordements - car il n'y avait aucun contrôle sur la foule. Certaines unités industrielles, telle la Société Tan Aliz, ont été détruites. À Bobo-Dioulasso, de nombreuses maisons ont été aussi saccagées, ainsi que l'hôtel de ville et le palais de justice.

Il est légitimement possible de comprendre les attaques contre les biens des dignitaires du régime tombé, même si les saccages et pillages enregistrés sont importants. Les propos entendus indiquent aussi que " les biens volés au peuple ont été repris par le peuple ». Autrement dit, les actes illégaux ont été justifiés par la légitimité de reprendre ce qui avait été volé. Certains ont même proposé que ce soit considéré comme des " frais collatéraux " inévitables. Lorsque le pouvoir a perdu toute légitimité pour avoir voulu ruser avec la loi, il n'y a plus que la légitimité de la rue qui prime.

\section{La confiscation de la révolution}

Vendredi 31 octobre, Blaise Compaoré a démissionné et il y avait vacance du pouvoir. C'est à ce moment que des leaders de la société civile, dont le Balai citoyen, ont demandé à l'armée de prendre ses responsabilités afin de restaurer la sécurité et d'arrêter le pillage. Le lieutenant-colonel Yacouba Isaac Zida a été désigné comme chef de l'État. Très vite les partis de l'opposition et les organisations de la société civile ont craint que « la révolution ait été confisquée » et le Balai citoyen fut accusé de trahison. Dimanche 2 novembre, il y avait une confusion totale sur la place de la Révolution, suivie d'un mouvement meurtrier à la télévision nationale. Dans cet après-midi, le lieutenant-colonel Zida a rencontré les représentants des partis politiques pour marquer le début de la transition. Le calme étant revenu, des solutions politiques ont été engagées et la communauté internationale s'est impliquée.

Néanmoins, toute la discussion sur la confiscation de la révolution reste d'actualité, nourrie par la nomination du lieutenant-colonel Zida comme premier ministre et l'implication de militaires dans le gouvernement. Pour que sa révolution ne soit pas confisquée, "la rue " reste vigilante. Dès l'annonce du gouvernement, elle a contraint à la démission un ministre qui 
avait été impliqué négativement dans l'instruction du dossier du journaliste assassiné Norbert Zongo. Quelques temps plus tard, le directeur de la Centrale d'achat des médicaments essentiels génériques (CAMEG) et le ministre des Infrastructures ont été contraints à la démission sous la pression de la contestation populaire. La vigilance des mouvements de la société civile - ou du moins de " la rue » - reste élevée. On a commencé à parler de "la ruecratie ", c'est-à-dire "la propension à manifester dans la rue pour exiger la résolution de certaines questions politiques » (Somda, 2015), suite à des remarques faites par le président Kafando. Si pour certains cela est de bonne guerre, pour d'autres, à force de recourir à la rue à tout va, on finira par perdre de vue l'essentiel.

\section{Le RSP : une armée dans l'armée}

Si le lieutenant-colonel Yacouba Isaac Zida a joui d'une légitimité comme chef de l'État pendant les deux semaines qui ont suivi la chute du régime Compaoré, sa nomination comme premier ministre du gouvernement de la transition pose problème. Le lieutenant-colonel Zida était numéro 2 du régiment de la sécurité présidentielle (RSP) sous Gilbert Diendéré, chef d'État-major particulier du président du Faso, chef des Renseignements généraux et main droite de Blaise Compaoré (Carayol et Roger, 2014 ; Carayol 2014). Dans le bimensuel burkinabè L'Evénement, Germain Bitiou Nama et Newton Ahmed Barry démontrent que quatre militaires ont agi vite pour ne pas perdre le pouvoir: Yacouba Isaac Zida, Honoré Nabéré Traoré, Djibril Bassolet et Gilbert Diendéré. Selon les révélations détaillées, il s'agit d'une "récupération pure et simple de l'insurrection par une partie de l'armée, notamment le RSP " avec l'objectif que "le pouvoir n'échappe pas au RSP " (Nama et Barry, 2014). Les luttes entre légalité et légitimité se heurtent ici à la force militaire, qui est partie intégrante du pouvoir burkinabè depuis 1966 (Hilgers et Loada, 2013). Le RSP a du reste montré sa force en obligeant le lieutenant-colonel Zida, par ailleurs ministre de la Défense, à ne pas le démanteler avant l'élection d'un président civil, arguant que cela n'était pas sa mission ${ }^{15}$. De même, le 4 février 2015 , des

15 Dans le journal l'Evénement du 10 janvier 2015, il est évoqué le fait que Zida voulait supprimer la prime de fin d'année octroyée aux soldats de ce régiment mais une révolution de 
éléments du RSP ont empêché la tenue d'un conseil des ministres pour protester contre des nominations en son sein. Cette crise majeure a sérieusement secoué la transition. En même temps, les manifestations de la rue ont clairement démontré la vigilance de la société civile et des partis politiques. En effet, elles ont demandé "la dissolution pure et simple du $R S P »$ (Boudani, 2015).

Au cœur de ces crises se situe la question de partage de pouvoir entre le président de la transition - et en même temps ministre des Affaires étrangères - Michel Kafando et le premier ministre de la transition - et simultanément ministre de la Défense - Yacouba Isaac Zida. La volonté déclarée d'en finir avec la corruption et de rouvrir les dossiers pendants du régime Compaoré pourrait être entravée par le fait que des éléments du RSP sont indexés dans beaucoup de crimes de sang commis, y compris l'assassinat de Norbert Zongo (Hagberg, 2002). En même temps, une lecture plus optimiste pourrait suggérer que seul quelqu'un comme le lieutenant-colonel Zida aurait la capacité et le pouvoir d'unir l'armée burkinabè. Alors, le rôle des forces armées reste une question clé pour le Burkina Faso post-Compaoré.

\section{La charte et la rue}

Actuellement (avril 2015), le Burkina Faso vit une situation où la Charte de la transition s'applique. L'élaboration de la Charte reste exemplaire et les Burkinabè en sont fiers. Des spécialistes en droit, tels que Luc lbriga et Augustin Loada, tous enseignants à l'université de Ouagadougou, ont activement contribué à donner une forme constitutionnelle légalement acceptable à la Charte. Mais tout n'est pas qu'une question de légalité car, audelà des institutions mises en place, "la rue " reste vigilante ${ }^{16}$. En effet, des nominations ont été dénoncées et ensuite annulées par « la rue ».

palais l'obligea à revenir sur sa décision. Zida a entamé le remplacement des responsables de cette garde présidentielle depuis novembre.

${ }^{16}$ A titre d'exemple, " la rue " a obligé les membres du conseil national de transition à revoir à la baisse leurs émoluments qui étaient jadis calqués sur ceux des députés de l'époque Compaoré. 
Cela révèle un autre problème du Burkina Faso post-Compaoré. Avec un méga-parti (tel le CDP), la mouvance présidentielle avec les partis accompagnateurs et aussi les associations de soutien à Blaise Compaoré (notamment la FEDAP/BC), presque " personne n'a été propre " sous l'ère Compaoré - de nos jours dénommé " compaorose " - qui a duré quasiment trois décennies! Donc, la purge qui se fait à travers les différentes nominations est nécessaire mais elle démontre aussi comment le régime de Blaise Compaoré cherchait à récupérer par des pratiques de corruption chaque tentative d'opposition et de contestation (Hilgers et Mazzocchetti, 2010). Aucune opposition ne pouvait émerger sans que les tentacules du pouvoir ne commencent à faire une "récupération politique et politicienne " du mouvement, du parti ou de l'acteur politique émergent en question. II restait donc incertain que "la rue " puisse persévérer si le légalisme politique occupait encore le devant de la scène.

Alors, toute une classe politique a été balayée d'un revers de la main. Au cours de la transition les institutions, acteurs et partis cherchent à revenir sur la scène publique. Le MPP de Roch March Christian Kaboré, Salif Diallo et Simon Compaoré semble assez fort mais, en même temps, la différence entre le CDP et le MPP est finalement une question de timing. Les gens du MPP ont su quitter le navire en janvier 2014 tandis que les gens du CDP sont restés. La légitimité se joue à deux niveaux ici : d'une part, le MPP et ses leaders faisaient partie de l'ancien système illégitime, d'autre part, ces personnalités ont une grande expérience de gouverner. En plus, même si des dirigeants du CDP et d'anciens ministres " circulent à Ouaga ", c'est surtout l'absence des plus aguerris du CDP qui est frappante. Mais, tôt ou tard, ils vont réapparaître et risquent de prendre du retard dans la course entreprise par les politiciens des autres partis. Si la légitimité est clamée par la rue, les partis politiques adjuvants de I'insurrection populaire semblent tous être déjà en campagne pour les élections de 2015. Le débat actuel sur la scène politique nationale se mène entre exclusion et inclusion, notions examinées à la lumière de la légalité et de la légitimité. 


\section{Conclusion}

Pour conclure cet article écrit à un moment où la transition est à michemin et où les acteurs et organisations de la société civile dénoncent différentes personnalités nommées aux postes de la transition, nous avons quelques remarques finales de cette ethnographie " à chaud ".

Premièrement, le bilan de cette insurrection est énorme en termes économiques et humains: entre 150 et 200 milliards de francs CFA de dégâts matériels divers, selon des chercheurs nationaux ${ }^{17}$. Selon le comité ad hoc commis à cette tâche, il y a eu 24 morts et 266 blessés sur l'ensemble du territoire. Plus tard ce chiffre a été modifié par la Coordination des structures pour l'assistance et le secours populaire (CAASP) avec 33 morts comptabilisés (Kaboré et Ouédraogo, 2014). Toutefois, pour de nombreux Burkinabè le résultat est positif et d'une valeur incommensurable en termes de transformation sociale et de renforcement de la démocratie au Burkina Faso et en Afrique. Le peuple burkinabè a donné une leçon pour l'histoire à tous ces dirigeants qui veulent s'éterniser au pouvoir en "tripatouillant la Constitution", selon l'expression consacrée. Les choses sont allées si vite que l'on a du mal à se rendre compte que Blaise Compaoré soit parti avant la fin de son mandat. Certains de ses anciens partisans demandent pardon au peuple, d'autres semblent encore un peu sonnés par la réalité.

Deuxièmement, la sortie massive des femmes sur la place publique, le 27 octobre a été une manifestation populaire assortie de symboles culturels. De façon paradoxale, ce qui aurait pu s'apparenter au signe d'un changement n'est rien d'autre que l'incarnation et la manifestation publique d'un rôle assigné socialement et culturellement. La clameur de la désobéissance civile des femmes atteste leur rôle de mères craignant un bain de sang à l'orée d'un pouvoir têtu, aveugle et sourd aux nombreux avertissements lancés par les partis de l'opposition et les acteurs de la société civile.

\footnotetext{
${ }^{17}$ L'institut Free Afric, une association dirigée par le Dr Rasablga Seydou Ouédraogo, a fait une étude rapide pour produire ce bilan qui concerne l'ensemble du pays.
} 
Troisièmement, tandis que le légalisme politique a été une arme utilisée pour développer une "démocratie à double façade ", la légitimité est devenue de plus en plus creuse, pour finalement aboutir à la chute de Blaise Compaoré. Cependant, un tel légalisme ne doit pas être confondu avec la légalité, car la révolution a largement concerné la défense de la loi fondamentale contre les abus du pouvoir. Après la révolution, des luttes entre légalité et légitimité semblent persister car, à chaque instant et à chaque tension, des décisions sont légitimées par rapport à la loi. Cependant, même si des luttes entre légalité et légitimité vont se poursuivre pendant la transition et après, il existe, dans la situation actuelle, des vraies potentialités pour bâtir une démocratie à la fois légitime et légale.

\section{Bibliographie}

AGENCE D'INFORMATION DU BURKINA (AIB), 2014a, "Burkina : Trois parties de la mouvance présidentielle soutiennent l'organisation d'un référendum ", $A I B, 18 / 09 / 2014$. Téléchargé le 8/1/ : http://www.aib.bf/m-1366-burkinatrois-partis-de-la-mouvance-presidentielle-soutiennent-l-organisation-dun-referendum.html

AGENCE D'INFORMATION DU BURKINA (AIB), 2014b, "Burkina : l'opposition appelle à une "désobéissance civile" à partir du 28 octobre ", $A / B$, 22/10/2014. Téléchargé le 26/11/2014: http://burkina24.com/2014 /11/25/insurrection-populaire-plus-de-600-blesses-et-19-morts-selon-uncomite-adhoc/

BARTHET E., 2014, "Six jours qui ont changé le Burkina Faso ", Le Monde.fr, $3 / 1 / 2014$. Téléchargé le $22 / 11 / 2014$ : http://www.lemonde.fr/ afrique/article/2014/11/03/six-jours-qui-ont-change-le-burkina-faso_ 4517145_3212.html\#z7JVZ2eRMoPyZcFO.99

BOUDANI Y ., 2015, "Burkina : la société civile dans la rue contre le RSP », Radio France Internationale, 7/02/2015. Téléchargé le 22 avril 2015 : http://www.rfi.fr/afrique/20150207-burkina-faso-compaore-regimentsecurite-presidentielle-societe-civile-rue/

CARAYOL R. et ROGER B., 2014, "Burkina : Il était une fois la révolution », Jeune Afrique, 2809, 9-15/11/2014. 
CARAYOL R., 2014, "Armée burkinabè : Gilbert Diendéré, la discrétion assurée ", Jeune Afrique, 5/11/2014. Téléchargé le 27/11/2014: http://www.jeuneafrique.com/Article/JA2711p056_059.xml0/

CHARTE DE LA TRANSITION, 13/11/2014. Téléchargé le 26/11/2014: http://lepays.bf/charte-transition-version-finale-adoptee/

CHOULI L., 2015, "The popular uprising in Burkina Faso and the Transition ", Review of African Political Economy, 42:144: 325-333

CONSTITUTION DU BURKINA, 1991, Adoptée par le Référendum du 02 juin 1991. Révisée par les lois constitutionnelles suivantes : $N^{\circ} 002 / 97 / A D P$ du 27 janvier 1997 ; N003-2000/AN du 11 avril 2000 ; N001-2002/AN du 22 janvier 2002. Téléchargé le 28/04/2015: http://ddata.over-blog.com /xxxyyy/1/35/48/78/Burkina-Faso/Constitution-consolidee-BurkinaFaso.pdf

FRERE M.S., 2010, « Enterrement de première classe ou leçon de droit ? La presse burkinabè et l'affaire Norbert Zongo ", in HILGERS M. et MAZZOCCHETTI J. (éds.), Révoltes et oppositions dans un régime semi-autoritaire : le cas du Burkina Faso, Paris, Karthala: 241-267.

FRERE M.-S. et ENGLEBERT P., 2015, "Briefing: Burkina Faso - the Fall of Blaise Compaoré ", African Affairs, 114(455) : 295-307.

FRINTZ A., 2014, "La jeunesse burkinabè bouscule la Françafrique ", Le Monde diplomatique, 729 : 8-9. Téléchargé le 21/2/2015 : http://www.mondediplomatique.fr/2014/12/FRINTZ/51057

GUE A., 2014, "Les héros de l'insurrection populaire ( $\left.{ }^{\circ} 9\right)$ : Cendrine Nama ", Lefaso.net, 27/11/2014. Téléchargé le 21/4/2015: http://www.lefaso.net/spip.php?article61993

HAGBERG S., 2002, "'Enough is Enough': An Ethnography of the Struggle against Impunity in Burkina Faso ", The Journal of Modern African Studies, 40 (2) : 217-246.

HAGBERG S., 2010, "Démocratie à double façade", in HILGERS M. et MAZZOCCHETTI J. (éds.), Révoltes et oppositions dans un régime semiautoritaire : le cas du Burkina Faso, Paris, Karthala : 295-306.

HAGBeRg S., 2014, "Is President Compaoré finally on the way out? ", Think Africa Press, 15 janvier 2014. Téléchargé le 15/1/2015 : http:// thinkafricapress.com/burkina-faso/enough-enough-compaore-long-rule 
HILGERS M. et LOADA A., 2013, "Tensions et protestations dans un régime semiautoritaire : croissance des révoltes populaires et maintien du pouvoir au Burkina Faso », Politique africaine, 131 : 187-208.

HILGERS M. et MAZZOCCHETTI J. (éds.), 2010, Révoltes et oppositions dans un régime semi-autoritaire : le cas du Burkina Faso, Paris, Karthala.

KABORE R.M.C. et al., 2014, " Lettre ouverte adressée à Monsieur Assimi Kouanda, Secrétaire Exécutif National du Congrès pour la Démocratie et le Progrès ", 4/01/2014.

KABORE A.F. et OUEDRAOGO S., 2014, " Insurrection populaire : la CAASP fait état de 33 morts ", Sidwaya, 3/12/2014. Téléchargé le 3/2/2015 : http://www.sidwaya.bf/index.php?I_nr=index.php\&l_nr_c=aeb764a6a85 4dd20beb97ec048c4ac14\&l_idpa=3551

LOUNGE M., 2014, « Insurrection populaire : Plus de 600 blessés et 19 morts selon un comité ad hoc ", Burkina24, 25/11/2014. Téléchargé le 26/11/2014 : http://burkina24.com/2014/11/25/insurrection-populaire-plus-de-600blesses-et-19-morts-selon-un-comite-adhoc/

LUNING S., 2010, « Réseau National de Lutte Anti-Corruption (REN-LAC) : A network for opposition ? ", in HILGERS M. et MAZZOCCHETTI J. (éds.), Révoltes et oppositions dans un régime semi-autoritaire: le cas du Burkina Faso, Paris, Karthala : 223-239.

NAMA G.B. et BARRY A.N., 2014, " Bassolet, Honoré, Gilbert et Zida : Comment ont-ils agi pour faire partir Blaise et ne pas perdre le pouvoir ", L'Evénement, $292: 8-9$.

OUESTAF NEWS, 2010, "Présidentielle au Burkina : Blaise fait un score comme au bon vieux temps, l'opposition proteste », 26/11/2010. Téléchargé le 10/5/2015 : http://www.ouestaf.com/Presidentielle-au-Burkina-Blaisefait-un-score-comme-au-bon-vieux-temps-l-oppositionproteste_a3371.html

PARE F., 2013, Assemblée nationale : Le projet de loi relatif au Sénat est adopté, Lefaso.net, 21/05/2013. Téléchargé le 5/1/2015 : http://www.lefaso.net/spip.php?article54290

PARE F., 2014, "Soutien de l'ADF-RDA à la modification de l'article 37 : "L'ADF-RDA a fait le choix de la paix et de la démocratie", dixit Gilbert Ouédraogo ", Lefaso.net, 25/10/2014. Téléchargé le 21 février 2015 : http://www.lefaso.net/spip.php?article61414 
SANFO A.D., 2014, "Le carton rouge de Hama Arba Diallo à Blaise Compaoré ", vidéo Youtube, 31/05/2014. Téléchargé 2/02/2015: https://www.youtube.com/watch?v=JOU_bQ1lAzo

SOMDA S., 2015, "Polémique sur la "ruecratie" : "On peut regretter qu'en quelques mois, Michel Kafando oublie d'où il vient", propos du Secrétaire général confédéral de la Confédération générale du travail du Burkina Bassolma Bazié », Lefaso.net, 13/01/2015. Téléchargé le 3/2/2015 : http://www.lefaso.net/spip.php?article62736

TRAORE A.E., 2014, " Marche-meeting de l'opposition : le gouvernement réagit ", Monfaso.info, 29/10/2014. Téléchargé le 22/2/2015: http://monfaso.info/index.php/faso/ouagadougou/1545-marchemeeting-de-I-opposition-le-gouvernement-reagit

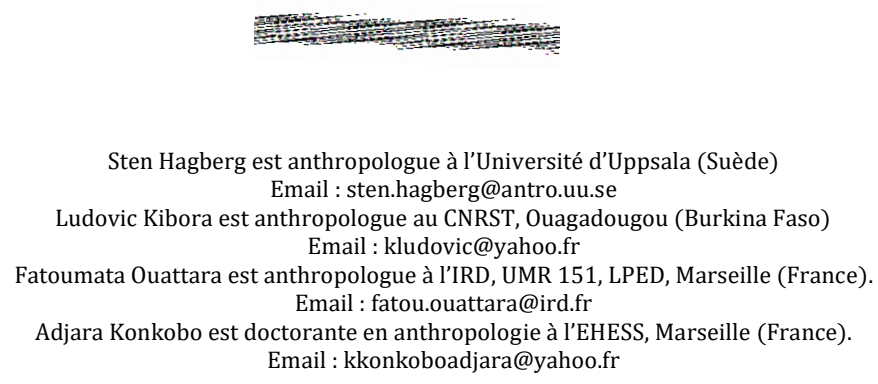

